\title{
Flow-mediated skin fluorescence: A novel method for the estimation of sleep apnea risk in healthy persons and cardiac patients
}

\author{
Tomasz Rechciński ${ }^{1}$, Urszula Cieślik-Guerra ${ }^{2}$, Patryk Siedlecki ${ }^{3}$, \\ Barbara Uznańska-Loch ${ }^{3}$, Ewa Trzos ${ }^{3}$, Karina Wierzbowska-Drabik ${ }^{1}$, Ewa Szymczyk ${ }^{1}$, \\ Paulina Wejner-Mik ${ }^{3}$, Małgorzata Kurpesa ${ }^{1}$, Piotr Lipiec ${ }^{1}$, Jarosław D. Kasprzak ${ }^{1}$ \\ ${ }^{1}$ Department of Cardiology, Medical University of Lodz, Poland \\ ${ }^{2}$ Department of Cardiac Rehabilitation, Bieganski Hospital, Lodz, Poland \\ ${ }^{3}$ Department of Cardiology, Bieganski Hospital, Lodz, Poland
}

\begin{abstract}
Background: A pilot study revealed a relationship between the results of flow mediated skin fluorescence (FMSF) and of ECG-Holter-based estimated apnea/hypopnea index (eAHI) in asymptomatic individuals. The aim of this study was to test whether the results of FMSF show a relationship with the eAHI in patients with coronary artery disease or aortic stenosis.

Methods: Twenty-one patients (12 coronary disease, 9 aortic stenosis) and 37 healthy volunteers were included. FMSF was assessed before, during and after the pressure occlusion of the brachial artery, using a prototype device allowing the quantification of skin fluorescence. The values of FMSF expressed as baseline (BASE), maximum (MAX), and minimum (MIN) were analyzed. The percentages of ischemic response (IR) and hyperemic response (HR) were calculated. The eAHI was assessed from night ECG-Holter recordings. Differences between the groups and the relationships between the parameters were analyzed statistically.

Results: Mean \pm standard deviation of BASE, MAX, MIN and IR were not significantly different in both groups $(p>0.05)$. HR was significantly lower in cardiac patients (14.7 \pm 7.5 vs. $11.8 \pm 5.1$; $p=0.048)$, whose eAHI was significantly higher $(11.0 \pm 7.4$ vs. $36.3 \pm 16.5$; $p<0.01)$. Negative correlation for MAX and eAHI was found in volunteers and patients: $r=-0.38, p=0.02$ and $r=-0.47$, $p=0.03$, respectively. In volunteers, HR had a negative correlation with eAHI: $r=-0.34, p=0.04$. Conclusions: This pioneer study confirms that FMSF can be used to detect the negative correlation between MAX fluorescence and eAHI not only among healthy volunteers, but also among cardiac patients with coronary artery disease or aortic stenosis. (Cardiol J 2022; 29, 6: 948-953)
\end{abstract}

Key words: autofluorescence, obstructive sleep apnea, screening

\section{Introduction}

Sleep disordered breathing has significant medical implications [1]. A reliable noninvasive method would be valuable for the screening of this condition in ambulatory patients. A pilot study with healthy volunteers revealed a promising potential for the assessment of flow mediated skin fluorescence (FMSF) in the selection of individuals with episodes of obstructive apnea during sleep [2]. FMSF is a method of assessing the redox balance in epithelial cells [3]. The question arises whether

Address for correspondence: Tomasz Rechciński, MD, PhD, Department of Cardiology, Medical University of Lodz, The Bieganski Hospital, ul. Kniaziewicza 1/5, 91-347 Łódź, Poland, tel: +48 4225161 12, e-mail: tomasz.rechcinski@office365.umed.pl

Received: 18.03.2020 Accepted: 13.10.2020 Early publication date: 26.10 .2020

This article is available in open access under Creative Common Attribution-Non-Commercial-No Derivatives 4.0 International (CC BY-NC-ND 4.0) license, allowing to download articles and share them with others as long as they credit the authors and the publisher, but without permission to change them in any way or use them commercially. 
nocturnal episodes of hypoxia caused by short periods of cessation of breathing can affect mitochondrial metabolism of the most superficial and distal organ such as skin - not only in healthy individuals, but also in cardiac patients, in whom the prevalence of obstructive sleep apnea is alarmingly high. The source of fluorescence (light of frequency $340 \mathrm{~nm}$ ) in this pilot study were the mitochondrial molecules of $\mathrm{NADH}-$ a reduced form of $\mathrm{NAD}^{+}$ (nicotinamide adenine dinucleotide) - after absorption of light energy emitted by the device Angiotester (Angionica Ltd., Lodz, Poland) [4]. The changes of the mitochondrial concentration of NADH are a consequence of hypoxia [5].

The aim of this study was to assess the relationship between the FMSF parameters and the values of the estimated apnea/hypopnea index (eAHI) in a group of healthy volunteers and cardiac patients with aortic stenosis or coronary artery disease $(\mathrm{CAD})$.

\section{Methods}

Thirty-seven healthy individuals were enrolled into the study. The inclusion criteria were:

- age 18-40 years;

- the absence of any treated or diagnosed chronic diseases;

- no antibiotics, pain relievers, or vaccinations during the prior 2 weeks;

- signing a written consent to participate in the study.

Twenty-one cardiac patients hospitalized at the Department of Cardiology at the Medical University of Lodz were included, using the following criteria:

- age > 40 years;

- diagnosis of CAD confirmed in coronary angiography showing stenoses $>70 \%$ or aortic stenosis with transaortic mean gradient $\geq 40 \mathrm{mmHg}$;

- completed treatment - surgical or transvascular;

- an obtained written consent for participation.

Patients with arrhythmia, after the implantation of devices for electrotherapy, with diagnosed sleep apnea syndrome or those reporting spontaneous symptoms suggesting sleep apnea were excluded.

All the participants signed a written consent before enrollment into the study. The protocol of the study was approved by the Local Ethics Committee at the Medical University of Lodz and was concordant with the Declaration of Helsinki.

Flow mediated skin fluorescence was measured on the forearm skin after 15-minute acclima- tization in a temperature-controlled room $(20 \pm$ $\pm 1^{\circ} \mathrm{C}$ ). First, the basic FMSF was measured for at least 2 minutes, followed by a measurement during a 100-second period of brachial artery occlusion and then again at least a 3-minute measurement was started. The flow of the blood in the left brachial artery was occluded by a cuff inflated around the arm up to $50 \mathrm{mmHg}$ above systolic blood pressure. The following parameters were measured: baseline fluorescence (BASE), maximum fluorescence (MAX) observed during occlusion, minimum fluorescence (MIN) after cuff release, and the following parameters were calculated: ischemic response (IR) defined as percentage difference between MAX and BASE (an increase in fluorescence intensity during occlusion), and hyperemic response (HR) defined as percentage difference between BASE and MIN (a decrease in intensity after the cuff release). Sample protocols of measurements performed in a healthy volunteer and in a cardiac patient are shown in Figures 1 and 2, respectively.

Coronary catheterization was performed according to the standard protocol using the INNOVA 2000 angiography system (General Electric). Two-dimensional echocardiography was performed by means of the (ViVid 7 device, GE), with the probe emitting ultrawaves of 2.5-4 MHz frequency according to the guidelines of the Working Group on Echocardiography of the Polish Cardiac Society. All patients and volunteers underwent a 24-hour electrocardiogram (ECG) Holter monitoring (Pathfinder 700, DelMar Reynolds, Hertford, UK). A three-channel recorder Lifecard CF was used. It was followed by further analysis with the Pathfinder 700 software and evaluation of the eAHI with Lifescreen Apnea (Spacelabs Healthcare, Issaquah WA, USA). An apneic event triggers the autonomic nervous system activation, which results in a specific sinus heart rhythm modulation. Lifescreen Apnea is a screening tool which calculates the probability of apneic events on the basis of changes in the beatto-beat intervals and the ECG-derived respiration signal. A detailed description of the method was given by de Chazal et al. [6].

A statistical analysis of mutual relationships between the eAHI and the parameters obtained from FMSF was performed using the Student t-test and correlation co-efficient with $\mathrm{p}$ value $<0.05$ regarded as significant.

\section{Results}

In the group of healthy volunteers there were $5(13.5 \%)$ cases of the eAHI above 15 , whereas 


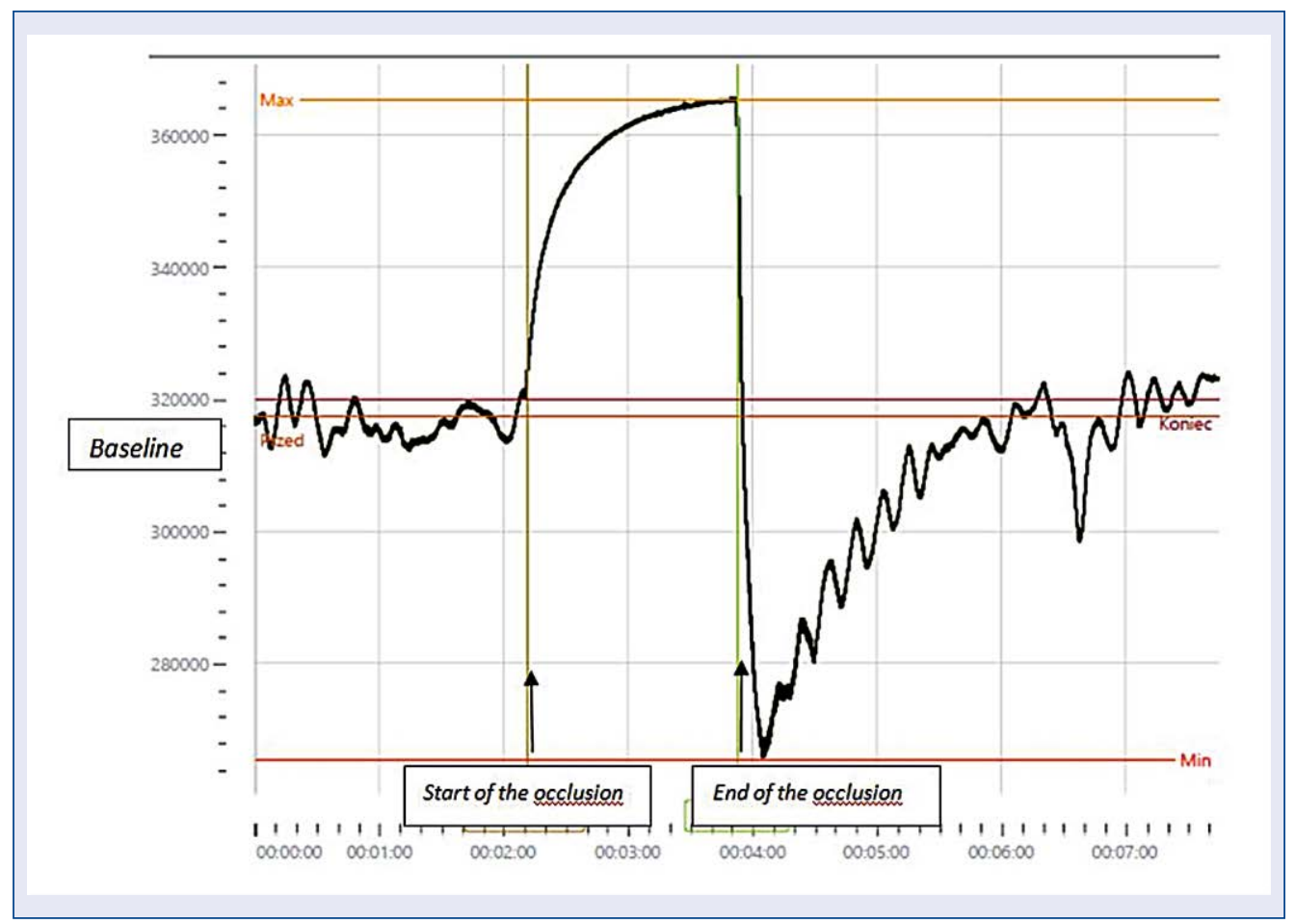

Figure 1. A sample protocol of measurement of flow mediated skin fluorescence in a healthy volunteer — baseline fluorescence (BASE) - 316200 arbitrary units, maximum fluorescence (MAX) - 364100, arbitrary units, minimum fluorescence (MIN) - 262400 arbitrary units, ischemic response $-15.1 \%$ and hyperemic response $-17.0 \%$; Przed — the beginning; Koniec — the end.

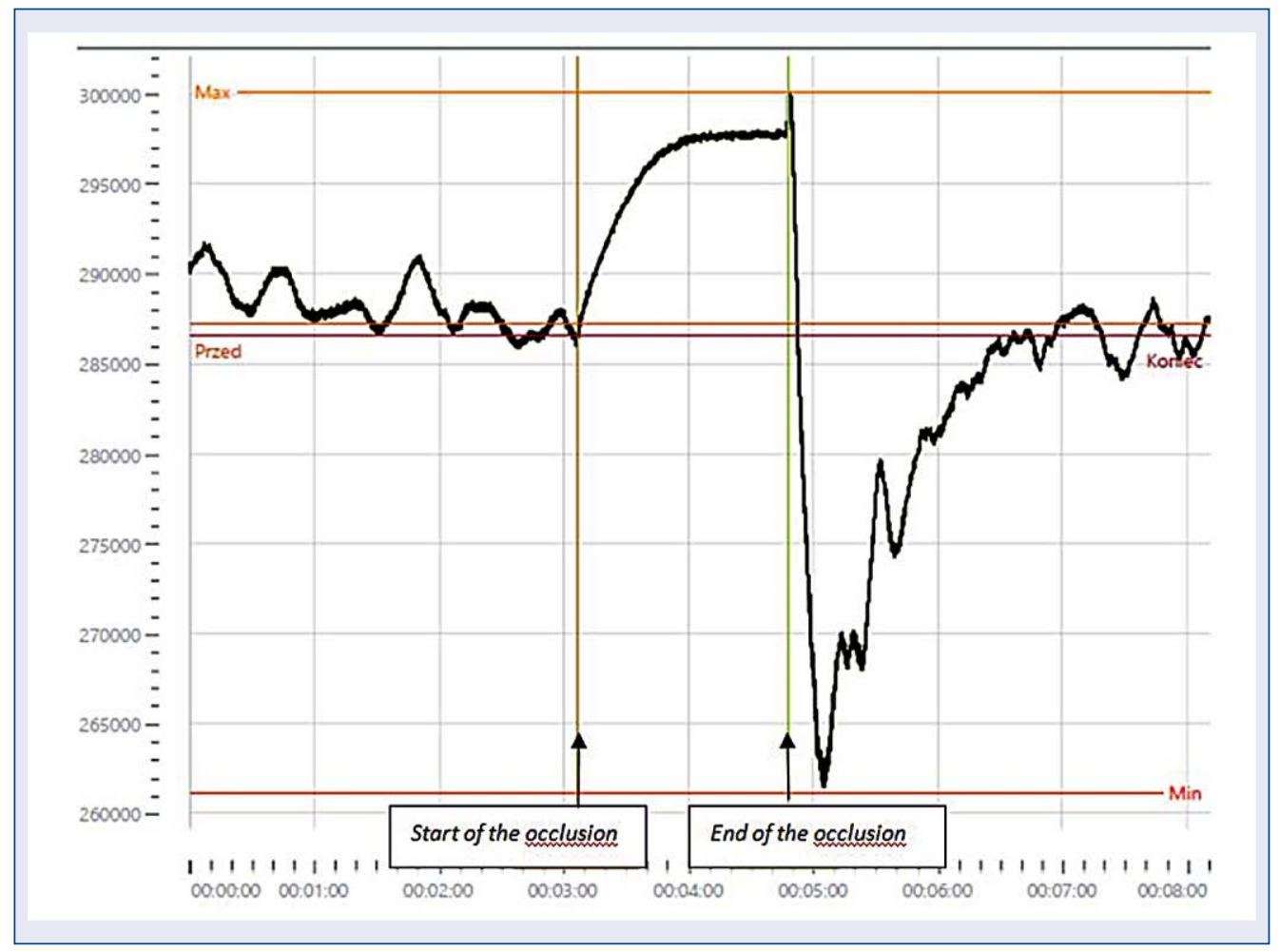

Figure 2. A sample protocol of measurement of flow mediated skin fluorescence in a cardiac patient — baseline fluorescence (BASE) - 286500 arbitrary units, maximum fluorescence (MAX) - 299400 arbitrary units, minimum fluorescence (MIN) - 261000 arbitrary units, ischemic response $-4.5 \%$ and hyperemic response $-8.9 \%$; Przed the beginning; Koniec — the end. 
Table 1. The clinical characteristics of the studied groups.

\begin{tabular}{lccc}
\hline Variable & $\begin{array}{c}\text { Healthy volunteers } \\
(\mathbf{n}=\mathbf{3 7})\end{array}$ & $\begin{array}{c}\text { Cardiac patients } \\
(\mathbf{n}=\mathbf{2 1})\end{array}$ & $\begin{array}{c}\text { Significance } \\
(\mathbf{P})\end{array}$ \\
\hline Age [years] & $32.9 \pm 5.2$ & $65.9 \pm 8.8$ & $<0.001$ \\
Sex, female & $12[32.4]$ & $7[33.3]$ & 1.0 \\
Body mass index & $26.13 \pm 2.9$ & $27.6 \pm 4.1$ & 0.065 \\
Systolic blood pressure [mmHg] & $125.62 \pm 9.38$ & $131.12 \pm 16.04$ & 0.002 \\
Diastolic blood pressure [mmHg] & $78.97 \pm 6.72$ & $80.57 \pm 12.48$ & $<0.001$ \\
Fasting glucose [mg/dL] & $89.56 \pm 8.06$ & $108.92 \pm 32.5$ & $<0.001$ \\
LDL-cholesterol [mg/dL] & $109.14 \pm 31.8$ & $99.1 \pm 38.7$ & 0.238 \\
Hemoglobin concentration [g/L] & $15.98 \pm 1.17$ & $13.71 \pm 1.54$ & $<0.001$ \\
Estimated apnea/hypopnea index & $11.0 \pm 7.4$ & $36.3 \pm 16.5$ & $<0.001$ \\
Left ventricular ejection fraction [\%] & $62.29 \pm 2.71$ & $51.07 \pm 8.21$ & $<0.001$ \\
Peak aortic jet velocity [m/s] & $1.26 \pm 0.11$ & $2.34 \pm 1.51$ & $<0.001$ \\
\hline
\end{tabular}

LDL — low-density lipoprotein

Table 2. Results of flow mediated skin fluorescence measurements.

\begin{tabular}{lccc}
\hline Variable & $\begin{array}{c}\text { Healthy volunteers } \\
(\mathbf{n}=\mathbf{3 7})\end{array}$ & $\begin{array}{c}\text { Cardiac patients } \\
(\mathbf{n}=\mathbf{2 1})\end{array}$ & $\begin{array}{c}\text { Significance } \\
(\mathbf{P})\end{array}$ \\
\hline $\begin{array}{l}\text { Baseline fluorescence } \\
{\left[\times 10^{3} \text { arbitrary units] }\right.}\end{array}$ & $360.2 \pm 122.9$ & $384.2 \pm 182.9$ & 0.505 \\
$\begin{array}{l}\text { Maximal fluorescence } \\
{\left[\times 10^{3} \text { arbitrary units] }\right.}\end{array}$ & $394.3 \pm 140.1$ & $395.1 \pm 206.1$ & 0.986 \\
$\begin{array}{l}\text { Minimal florescence } \\
{\left[\times 10^{3} \text { arbitrary units] }\right.}\end{array}$ & $300.5 \pm 103.4$ & $336.3 \pm 206.0$ & 0.240 \\
Ischemic response [\%] & $2.4 \pm 9.8$ & $0.15 \pm 4.9$ & 0.176 \\
Hyperemic response [\%] & $14.7 \pm 7.5$ & $11.8 \pm 5.1$ & 0.048 \\
\hline
\end{tabular}

among cardiac patients there were $18(85.7 \%)$ such cases.

The comparison of characteristics of volunteers and cardiac patients is presented in Table 1. All the cardiac patients received beta-blockers, angiotensin-converting enzyme inhibitors and statins, $12(57.1 \%)$ patients were on antidiabetic medication.

No significant differences were found between the values of basic, maximal, and minimal fluorescence, and in the IR between the cardiac patients and volunteers; however, HR was significantly higher in the volunteers $-14.7 \pm 7.5$ vs. $11.8 \pm 5.1$ $(\mathrm{p}=0.048)$. The detailed data are presented in Table 2 . Interestingly, despite similar average fluorescence values between the two investigated groups, significant negative correlation coefficients were found between the values of maximal fluorescence and the eAHI in both groups. Additionally, significant negative correlation was found for HR and the eAHI in healthy volunteers (Table 3 ).
Table 3. The correlations between flow mediated skin fluorescence and the estimated apnea/ /hypopnea index (eAHI).

\begin{tabular}{lcc}
\hline $\begin{array}{l}\text { Correlated } \\
\text { variables }\end{array}$ & $\begin{array}{c}\text { Healthy } \\
\text { volunteers }\end{array}$ & $\begin{array}{c}\text { Cardiac } \\
\text { patients }\end{array}$ \\
\hline $\begin{array}{l}\text { Maximal } \\
\text { fluorescence }\end{array}$ & $\mathrm{r}=-0.38$ & $\mathrm{r}=-0.47$ \\
$\begin{array}{l}\text { and eAHI } \\
\text { Hyperemic }\end{array}$ & $\mathrm{p}=0.02$ & $\mathrm{p}=0.03$ \\
$\begin{array}{l}\text { response } \\
\text { and eAHI }\end{array}$ & $p=0.34$ & $r=-0.07$ \\
\hline
\end{tabular}

\section{Discussion}

The main finding of the present study is that the severity of sleep apnea assessed by means of the eAHI derived from 24-hour Holter monitoring is correlated with noninvasive measurements of redox parameters obtained in vivo in a simple rapid test, i.e., FMSF. This was observed both in healthy volunteers and in cardiac patients. 
This finding is especially practical in the context of very high prevalence of abnormal eAHI in cardiac patients, in the absence of self-reported sleepiness or the presence of risk factors for sleep-disordered breathing. This observation is concordant with the results of previous studies by Ben Ahmed et al. [7] and by Mehra et al. [8], and it underlines a strong need for reliable and easy tools for sleep apnea screening.

The reference method - polysomnography - is a costly procedure, time-consuming and less available, and therefore it cannot be used as a screening test. Therefore, Holter monitoring is often proposed for first-step diagnostics of sleep apnea. Recent publications confirm the reliability of a simpler diagnosis of obstructive respiratory disorders on the basis of 24-hour Holter monitoring by means of the Lifescreen Apnea software making use of eAHI in various groups of patients for screening purposes. This approach was used in our study and it was observed previously that the cut-off value of eAHI set at 17 was optimal for the differentiation between patients with or without sleep-related breathing disorders $[9,10]$. The existing evidence confirms the repeatability of the results of Holter-based eAHI derived from two consecutive nights on the basis of 48-hour ECG monitoring [11]. The critical advantage of FMSF over 24-hour Holter monitoring as a screening tool for sleep apnea is the much shorter duration of the test, which takes only 25 minutes.

The application of FMSF for the detection of abnormal microvascular function in healthy controls and patients with stable CAD was already described by Hellmann et al. [12]. Those authors reported both the excellent reproducibility of FMSF examination performed day by day and a significantly lower IR and HR when cardiac patients were compared with healthy volunteers. The same relationship was found in the present groups, but the difference was significant only for HR. The next study of that team, published by Tarnawska et al. [13], emphasizes a significant inverse correlation of HR with the blood concentration of ADMA (asymmetric dimethyl arginine - endogenous competitive inhibitor of nitric oxide synthase), and of IR with the concentration of endothelin-1 - vasoconstrictor and mitogen produced in response to hypoxia and vascular wall stress [13]. Nevertheless, the problem of night periods of hypoxia due to sleep apnea was not investigated in that previous study and the current report seems to be the first in this field of investigations.
The potential impact of night periods of hypoxia on the status of mitochondria on the following morning was described by two independent teams - Lacedonia et al. [14] and Kim et al. [15] - who found that people with obstructive sleep apnea have a higher concentration of damaged mitochondrial DNA than people without this disease. Since mitochondria are the only organelle where $\mathrm{NADH}$ - the source of fluorescence - is present, and since they have limited DNA repair capacity, impaired mitochondrial function could explain the linkage between sleep apnea and the lower values of HR observed with FMSF in the individuals participating in the present study.

The lack of polysomnography to diagnose sleep apnea and the relatively small groups participating in the study should be regarded as the main limitation of this study. Further investigations of this phenomenon on a larger sample of participants and with the use of methods directly assessing sleep apnea should be performed.

\section{Conclusions}

This pioneer study confirmed in a relatively small study group that a noninvasive test - FMSF can be used to detect a negative correlation between maximum FMSF and the eAHI not only among healthy volunteers, but also among cardiac patients with CAD or aortic stenosis.

\section{Funding}

The study was performed within the framework of the project "Novel technique for assessing microvascular circulation: Flow-Mediated Skin Fluorescence. Constructing a prototype of the device and its clinical verification" and it was supported from European Funds - project no. POIR.01.01.01-00-0540/15. All authors received remuneration from this project.

\section{Conflict of interest: None declared}

\section{References}

1. Dredla BK, Castillo PR. Cardiovascular consequences of obstructive sleep apnea. Curr Cardiol Rep. 2019; 21(11): 137, doi: 10.1007/s11886-019-1228-3, indexed in Pubmed: 31707504.

2. Rechciński T, Cieślik-Guerra U, Siedlecki P, et al. Flow-mediated skin fluorescence - a novel screening tool for cardiovascular risk. Eur Heart J. 2018; 39(Suppl 899): P4459.

3. Mayevsky A, Barbiro-Michaely E. Shedding light on mitochondrial function by real time monitoring of NADH fluorescence: II: 
human studies. J Clin Monit Comput. 2013; 27(2): 125-145, doi: 10.1007/s10877-012-9413-6, indexed in Pubmed: 23224276.

4. Piotrowski L, Urbaniak M, Jedrzejczak B, et al. Note: flow mediated skin fluorescence: a novel technique for evaluation of cutaneous microcirculation. Rev Sci Instrum. 2016; 87(3): 036111, doi: 10.1063/1.4945044, indexed in Pubmed: 27036844.

5. Sibrecht G, Bugaj O, Filberek P, et al. Flow-mediated skin fluorescence method for non-invasive measurement of the NADH at $460 \mathrm{~nm}$ - a possibility to assess the mitochondrial function. Post Biol Kom. 2017; 44(4): 333-352.

6. de Chazal P, Heneghan C, Sheridan E, et al. Automated processing of the single-lead electrocardiogram for the detection of obstructive sleep apnoea. IEEE Trans Biomed Eng. 2003; 50(6): 686-696, doi: 10.1109/TBME.2003.812203, indexed in Pubmed: 12814235 .

7. Ben Ahmed H, Boussaid H, Hamdi I, et al. [Prevalence and predictors of obstructive sleep apnea in patients admitted for acute myocardial infarction]. Ann Cardiol Angeiol (Paris). 2014; 63(2): 65-70, doi: 10.1016/j.ancard.2014.01.003, indexed in Pubmed: 24485826.

8. Mehra R, Principe-Rodriguez K, Kirchner HL, et al. Sleep apnea in acute coronary syndrome: high prevalence but low impact on 6-month outcome. Sleep Med. 2006; 7(6): 521-528, doi: 10.1016/j.sleep.2006.03.012, indexed in Pubmed: 16931151.

9. Szyszko A, Franceschini C, Gonzalez-Zuelgaray J. Reliability of a Holter-based methodology for evaluation of sleep apnoea syndrome. Europace. 2009; 11(1): 94-99, doi: 10.1093/europace/ /eun285, indexed in Pubmed: 18971289.

10. Ożegowski S, Wilczyńska E, Piorunek T, et al. Usefulness of ambulatory ECG in the diagnosis of sleep-related breathing disorders. Kardiol Pol. 2007; 65(11): 1321-8.

11. Uznańska B, Trzos E, Rechciński T, et al. Repeatability of sleep apnea detection in 48-hour holter ECG monitoring. Ann Noninvasive Electrocardiol. 2010; 15(3): 218-222, doi: 10.1111/j.1542474X.2010.00367.x, indexed in Pubmed: 20645963.

12. Hellmann M, Tarnawska M, Dudziak M, et al. Reproducibility of flow mediated skin fluorescence to assess microvascular function. Microvasc Res. 2017; 113: 60-64, doi: 10.1016/j. mvr.2017.05.004, indexed in Pubmed: 28529171.

13. Tarnawska M, Dorniak K, Kaszubowski M, et al. A pilot study with flow mediated skin fluorescence: A novel device to assess microvascular endothelial function in coronary artery disease. Cardiol J. 2018; 25(1): 120-127, doi: 10.5603/CJ.a2017.0096, indexed in Pubmed: 28840593.

14. Lacedonia D, Carpagniano GE, Cisetti E, et al. Mitochondrial DNA alteration in obstructive sleep apnoea. Resp Res. 2015; 16: 47.

15. Kim YS, Kwak JW, Lee KE, et al. Can mitochondrial dysfunction be a predictive factor for oxidative stress in patients with obstructive sleep apnea? Antioxid Redox Signal. 2014; 21(9): 1285-1288, doi: 10.1089/ars.2014.5955, indexed in Pubmed: 24926527. 